\title{
Responsabilidad ciudadana en la prevención de drogas: un estudio desde las redes sociales ${ }^{1}$
}

Institución: Universidad de Costa Rica

\author{
Viriam Leiva Díaz ${ }^{2}$ \\ Lucía Cordero Villanueva ${ }^{3}$ \\ Elena Loría Varela ${ }^{4}$ \\ Fernanda Morales Alvarado ${ }^{5}$ \\ Jessica Vega Mejías ${ }^{6}$
}

\section{COMO CITAR}

\begin{abstract}
Leiva, V. ,Cordero, L., Loría, E., Morales, F., Vega, J. (2014). Responsabilidad ciudadana en la prevención de drogas: un estudio desde las redes
\end{abstract} sociales. Rev. Enfermería Actual en Costa Rica, 27, 1-17. DOI: http://dx.doi.org/10.15517/revenf.v0i27.16024

\section{RESUMEN}

Introducción. El objetivo de este artículo es presentar los resultados del estudio sobre la responsabilidad ciudadana en la prevención del fenómeno de las drogas, la cual debe caracterizarse por ser una participación activa y solidaria de parte de cada uno de los miembros de la sociedad costarricense.

Método. La investigación es de tipo exploratoria, con un enfoque cuantitativo, de corte transversal. La población está constituida por 353 habitantes de Costa Rica inscritos en la red social Facebook. Para recolectar los datos, en dicha red social se formuló preguntas generadoras a manera de encuesta.

Resultado. Dentro de los hallazgos se puede mencionar que las personas participantes conocen todos los aspectos que se encuentran inmersos en el concepto de responsabilidad ciudadana, sin embargo, existe una gran evasión, por parte de los pobladores, respecto de la prevención de las drogas.

Conclusión. Los y las participantes no ejercen su responsabilidad ciudadana por desconocimiento, sea de leyes o de las políticas públicas, delegando dicha responsabilidad a diferentes entes, personas o instituciones.

Palabras clave: ciudadanía; enfermería; prevención-de-drogas; redes-sociales; responsabilidad.

\footnotetext{
${ }^{1}$ Fecha de recepción:

Fecha de aceptación:

2 Escuela de Enfermería, Universidad de Costa Rica. Costa Rica. Correo electrónico: viriaml@gmail.com

3 Enfermera. Labor independiente. Costa Rica. Correo electrónico: lucia villanueva86@hotmail.com

${ }^{4}$ Enfermera. Labor independiente. Costa Rica. Correo electrónico: elyalova@hotmail.com

${ }^{5}$ Escuela de Enfermería, Universidad de Costa Rica. Costa Rica. Correo electrónico: mfermorales89@gmail.com

${ }^{6}$ Enfermera. Labor independiente. Costa Rica. Correo electrónico: jvegamejias@gmail.com
} 


\section{Civic responsibility in drug prevention: a study from social networks ${ }^{1}$}

Institution: University of Costa Rica

Viriam Leiva Díaz ${ }^{2}$

Lucía Cordero Villanueva ${ }^{3}$

Elena Loría Varela ${ }^{4}$

Fernanda Morales Alvarado ${ }^{5}$

Jessica Vega Mejías ${ }^{6}$

\section{CITED}

Leiva, V. ,Cordero, L., Loría, E., Morales, F., Vega, J. (2014). Civic responsability in drug prevention: a study from social networks. Rev. Enfermería Actual en Costa Rica, 27, 1-17. DOI: http://dx.doi.org/10.15517/revenf.v0i27.16024

\section{ABSTRACT}

Background: the aim of this article presents the results of the study on civic responsibility in preventing drug phenomenon, this being an active and supportive participation of each of the members of the Costa Rican society, research was exploratory under the quantitative approach of cross section.

Method: the population consisted of 353 people registered inhabitants of Costa Rica on the social network Facebook. To collect the data generating way to survey questions on that page were used.

Result: Among the findings it can be mentioned that participants know all the aspects that are embedded in the concept of civic responsibility, however, there is a great escape, by the people, to this in terms of drug prevention. Conclusion: The participants do not exercise their civic responsibility through ignorance, whether of law or public policy, delegating that responsibility to different entities, individuals or institutions.

Key words: citizenship; drug-prevention; nursing; responsibility; social-networks.

\footnotetext{
${ }^{1}$ Date of receipt:

Date of acceptance:

2 Escuela de Enfermería, Universidad de Costa Rica. Costa Rica. Correo electrónico: viriaml@gmail.com

3

${ }^{3}$ Nurse. Independent work. Costa Rica. Correo electrónico: lucia_villanueva86@hotmail.com

${ }^{4}$ Nurse. Independent work. Costa Rica Correo electrónico: elyalova@hotmail.com

${ }^{5}$ Escuela de Enfermería, Universidad de Costa Rica. Costa Rica. Correo electrónico: mfermorales89@gmail.com

6 Nurse. Independent work. Costa Rica. Correo electrónico: jvegamejias@gmail.com
} 


\section{INTRODUCCIÓN}

El ser humano que vive en sociedad tiene derechos y deberes consigo y la sociedad, los cuales son individuales y colectivos (Beck, 2007), de modo que se establece una diferencia entre la responsabilidad individual y la social; ambos tipos de responsabilidad son parte de los deberes que corresponden al ser humano, como individuo y ciudadano (a) o miembro de una comunidad (Constitución Política de Costa Rica, artículo 1, 1949).

Respecto del concepto de ciudadanía, Cortina (1997) lo presenta desde diferentes perspectivas, como la política, civil, económica e intercultural y social. En cuanto a esta última, la autora define al ciudadano como

"el que se ocupa de las cuestiones públicas y no se contenta con dedicarse a sus asuntos privados, sino que además es quien sabe que la deliberación es el procedimiento más adecuado para tratarlas, más que la violencia, más que la imposición” (p. 66).

Como lo señala Cortina (1997), para ejercer un verdadero papel de ciudadano o ciudadana lo más importante es demostrar una responsabilidad ciudadana activa, en la cual se trabaje a partir de la comunicación y la participación social. La trascendencia de la participación social radica en su aporte respecto de la construcción de una sociedad activa y creciente; es decir tal como lo afirma Gamboa (2007), la comunidad política y social- se consolida en la participación responsable y se fortalece por la pertenencia a una sociedad civil y solidaria, entonces, no se puede lograr su crecimiento, si sus individuos no participan activamente dentro de todos los procesos.

Definido lo anterior, se requiere que la ciudadanía sea ejercida por las personas de manera responsable; por tanto, es necesario que se desarrolle una conciencia de lo que implica. Sin embargo, como lo expresa Cuadra (2001), propiciar una cultura de responsabilidad ciudadana también es un reto para diferentes miembros de la sociedad, tales como comunicadores, educadores y padres, quienes deben saber cómo aflora el conflicto para enfrentarlo y resolverlo antes de que llegue al estado de crisis.

De este modo, es vital para el desarrollo del país que los actores sociales intervengan en pro del mejoramiento de la calidad de vida de los y las ciudadanas (Dirección del Triángulo de la Solidaridad, 2000). La legislación costarricense incluye algunos espacios para que la sociedad participe en diferentes temas y actividades de la vida nacional, los cuales permiten que estos ejerzan sus derechos y deberes, los cuales en el nivel local se refieren a juntas de educación, asociaciones de desarrollo, juntas de protección a la infancia pertenecientes al Patronato Nacional de la Infancia (PANI), comités de salud, entre otros.

Como se observa, la responsabilidad social es un valor importante para lograr la participación ciudadana (OMS, 2006), puesto que, al practicar este valor, se fomenta un interés real en torno a los escenarios que enfrenta la sociedad.

Una de las situaciones que afronta la ciudadanía actualmente es el fenómeno de las drogas, un problema global que está en constante incremento. Al respecto, la OMS (2006) expresa que las drogas repercuten en la sociedad y generan una gama de problemas familiares, sociales e individuales, tanto en países desarrollados como 
en vías de desarrollo. Así, su uso se convierte en un problema creciente en términos de salud pública y pone en evidencia que la ésta y la responsabilidad social están interrelacionadas, dado que los problemas de la primera repercuten en la segunda y viceversa, entonces, al ejercer la responsabilidad social se pueden resolver algunos problemas de salud pública.

Consciente de dicha interrelación, desde la disciplina enfermera, es imprescindible fortalecer el valor de la responsabilidad social, debido a que es una profesión que enmarca a la persona dentro del enfoque de los determinantes sociales y de la salud (OMS, 2000). Partiendo de lo anterior, se afirma que la responsabilidad social es de gran importancia al trabajar con los sujetos, debido a que la promoción de la salud brinda herramientas para mejorarla o mantenerla en un estado aceptable; sin embargo, las acciones tomadas para este fin son responsabilidad de la población, por ende, reconocer dicho valor facilita la construcción de una realidad social actual sobre el tema, punto que se constituye en el objetivo de este estudio.

De lo anterior, surge la pregunta, ¿cuáles responsabilidades tienen los ciudadanos en cuanto a la prevención del fenómeno de las drogas? Para reflexionar al respecto, se debe partir de que a cada miembro de una sociedad le concierne múltiples obligaciones, tal como se menciona en la Ley General de Salud de Costa Rica (Ministerio de Salud, 1973), "toda persona debe velar por el mejoramiento, la conservación y la recuperación de su salud personal y la de los miembros de su hogar y comunidad" (art. 9); dentro de estas se encuentra la prevención del fenómeno de las drogas.

\section{MATERIALES Y MÉTODO}

En cuanto a la población, se limitó a todo habitante de Costa Rica inscrito en la red social Facebook, que ingresara un comentario en el grupo creado por las investigadoras para este fin, el cual se llamó A usted, ¿le importa? La recolección de datos fue durante un periodo de tres meses establecido.

La población estuvo constituida por 353 participantes que recibían toda la información y preguntas generadas en la página; no obstante, solamente un sector de esta población respondió cada propuesta (un rango entre 40 a 50) debido, por una parte, al respeto del principio de autonomía que cada usuario posee y, por otra, al tiempo limitado en que se publicaba las interrogantes.

La investigación realizada fue de tipo exploratorio, dado que el objetivo fue examinar un tema de investigación poco estudiado, el cual es la responsabilidad ciudadana en la prevención de drogas; desde un enfoque cuantitativo (Hernández, Fernández y Baptista, 2007).

Para asegurar la rigurosidad metodológica, fue fundamental el desarrollo del sistema de variables, tales como: datos sociodemográficos, responsabilidad ciudadana, prevención de drogas.

Dentro de la página se publicaron diversas preguntas generadoras por medio de la técnica de encuesta, cuyo fin es motivar la discusión entre los participantes. Dichas preguntas fueron validadas según el criterio de ocho jueces, familiarizados con las variables o afines al tema. 


\section{Fases de la investigación}

Estas fases permiten explicar las acciones realizadas dentro del espacio virtual Facebook, acción fundamental debido a que la herramienta utilizada para formular las preguntas es innovadora y poco común en el campo de la enfermería.

En relación con lo anterior, seguidamente se explica el procedimiento llevado a cabo.

\section{Fase I: Apertura del espacio virtual}

En primer lugar, se abrió un espacio virtual, por medio de una cuenta en la red social Facebook, en la modalidad de organización sin fines de lucro y perfil. El nombre de dicho espacio fue $\boldsymbol{A}$ usted, ¿le importa? y en él las personas que por afinidad desearan contestar, comentar u opinar sobre el tema, podían hacerlo de forma libre.

La red social Facebook es un sitio web gratuito en el que todo aquel que cuente con un correo electrónico puede inscribirse y formar parte de él (Flores, 2009). Para ello, se ingresa varios datos personales que la página solicita (en la columna derecha), tales como nombre, apellidos, edad, nombre de usuario, correo electrónico, una contraseña creada por el usuario, país, ciudad de origen y ciudad de procedencia, información valiosa para el estudio.

De igual manera, es posible crear una página que, para este caso específico, se abrió en la parte de organización sin fines de lucro, la cual funciona como un perfil, pero es sobre un tema específico y las personas que gusten podrán unirse, opinar, adjuntar fotografías, videos, entre otros. Además, dicha página cuenta con la característica de que su administrador puede limitar la población de ingreso con respecto a la edad, lugar de residencia, sexo, entre otros, logrando de esta manera filtrar la información recibida.

\section{Fase II: Promoción del espacio}

Una vez creado el espacio virtual, fue fundamental su divulgación, con el objetivo de atraer la mayor cantidad de personas. Para ello, se utilizaron medios virtuales como el correo electrónico, para enviar un enlace a la página con el que cada propietario de un correo electrónico ingresa. Además, Facebook cuenta con una opción de sugerir a los amigos distintos enlaces y páginas, recurso aprovechado para enviar sugerencias a distintas personas con el fin de divulgar el espacio; aparte, de que se promocionó la página A usted, ¿le importa? en los muros de páginas de interés público dentro de la misma red social, por ejemplo, en las de Teletica Canal 7 , Universidad de Costa Rica, Universidad Nacional, Instituto Tecnológico de Costa Rica, Emisoras de Radio, entre otras.

Con el mismo objetivo de promover la página de Facebook, se realizó una distribución de aproximadamente 500 volantes, con el logo y nombre de la página, así como la fecha correspondiente al inicio de la exposición de las preguntas y diversa información acerca del estudio. 


\section{Fase III: Uso del servicio de Facebook}

Para efectos de este estudio, se utilizó los principales servicios que ofrece la red social Facebook, de la siguiente manera:

a) Se formó un grupo llamado A usted, ¿le importa?, el cual contó con una serie de vínculos como lo son: - Información, incluye datos generales del grupo y su finalidad de creación, además, se localiza una dirección electrónica a la cual los usuarios pueden referirse, así como el consentimiento informado.

- Muro, espacio en el que se publica las preguntas generadoras de la investigación, además, ahí las personas pueden realizar sus comentarios a partir de las preguntas formuladas. La cantidad de comentarios de un mismo usuario no está restringida ni la discusión entre usuarios. En este apartado se encuentra el Estado, en el que, específicamente, se editaron y publicaron las preguntas generadoras. En esa parte, se puede observar una vista miniatura de la imagen o logo que representa al grupo, así como la lista de sus amigos.

- Fotos y videos en la cual se adjuntan fotografías o videos alusivos al tema para generar interés de los usuarios y usuarias.

Dentro de estas aplicaciones se encuentra la correspondiente a Preguntas, la cual ofrece a los participantes colocar una respuesta y, en caso de estar de acuerdo con alguna opción ya publicada, marcar dicha opción y votar por ella. Este sistema permite agrupar las repuestas similares de manera automática.

\section{Fase: Publicación de preguntas generadoras}

Las preguntas generadoras se brindaron en el espacio de Facebook llamado Estado, el cual permitió a los usuarios y usuarias expresar pensamientos, emociones u otro asunto personal que desearan compartir con los demás miembros.

Las preguntas fueron brindadas de forma aleatoria y dependiendo de la dinámica de los usuarios; cada pregunta tenía un tiempo limitado de exposición al público, luego de este se eliminaba y se publicaba otra de acuerdo con el criterio de las investigadoras.

Se revisó el espacio virtual aproximadamente dos veces al día, de manera que se extrajo todos los comentarios y se revisó los perfiles de los usuarios.

\section{Consideraciones éticas}

Un aspecto esencial del trabajo fue que, durante su realización (tanto en la fase de elaboración como en la de ejecución), se tomó en cuenta y se aplicó los principios bioéticos que deben ser empleados en toda investigación con personas (Blazques, 2004, CIOMS, 2002), entre los cuales se incluyó:

-La autonomía: consiste en reconocer el derecho de todo participante a realizar comentarios o no dentro del perfil; retirarse en cualquier momento; abstenerse de hacer comentarios; tener sus propios puntos de vista; adjuntar 
cualquier archivo (video, fotografía, documento, entre otros) alusivo a las temáticas por tratar en el espacio, sin embargo, no se permitió contenidos pornográficos u ofensivos para cualquier grupo social, étnico, político, religioso, entre otros. Finalmente, actuar en conformidad con valores y creencias de cada persona.

-La privacidad: se mantuvo el anonimato de los sujetos del estudio y en ningún momento se reveló sus nombres u otros detalles no pertinentes a la investigación.

-El consentimiento informado: este proceso se realizó brindando información a los participantes sobre la dinámica del estudio, el cual finalizó con la publicación de los comentarios de los sujetos, a modo de firma de autorización, tal como se explicó en el consentimiento escrito. Debido a las características propias del espacio virtual, el proceso del consentimiento informado se hizo a través de la mediación virtual y se concretó dentro de la información general del grupo.

\section{RESULTADOS}

En este apartado se ofrecen los resultados de las variables correspondientes a datos sociodemográficos, responsabilidad ciudadana y prevención de drogas.

El total de la población partícipe fue de 353 personas inscritas, de las cuales un 57\% corresponde a mujeres y un 27\% a hombres; un 16\% impidió el acceso a información de este tipo, debido a la privacidad de su perfil.

En cuanto a la categoría edades, el grupo etario mayoritario fue el de personas de 18 a 24 años (48\%), seguido por el de 13 a 17 años (36\%). Un 16\% corresponde a sujetos, cuya edad exacta no fue posible determinar, por las características propias de la red social.

Conocer el concepto que maneja la población de estudio sobre la responsabilidad ciudadana tiene una importancia significativa para el desarrollo de los objetivos del estudio, dado que permite crear un panorama general sobre los conocimientos y las formas de pensar de los protagonistas sociales, capaz de explicar, en alguna medida, su actuar en una comunidad determinada. A partir del planteamiento anterior, y respecto de la responsabilidad ciudadana, cabe mencionar que la mayoría de los participantes concuerdan en que es parte de las obligaciones que cada persona tiene como miembro de una sociedad.

Las definiciones brindadas por los participantes se relacionaban entre sí y posibilitaban identificar cómo la población de estudio conceptúa la responsabilidad ciudadana, acerca de lo que mencionaron la necesidad de un compromiso que debe marcar una pauta en el ejercicio de la ciudadanía, es decir, puede influir de manera significativa para que esta se ejerza de una forma activa, de modo que los ciudadanos y ciudadanas sean más que unos espectadores con voto.

Al formar parte de una sociedad, un ciudadano requiere ser partícipe en las decisiones de asuntos comunitarios, interés que se refleja en el accionar real del sujeto en cada asunto, lo cual incluye las necesidades que la sociedad posea que, en este caso, se refiere a la prevención de drogas. Dado lo anterior, es necesario evaluar el ejercicio de la responsabilidad frente a dicho fenómeno, información que se muestran en la tabla 1 


\author{
Tabla 1 \\ Escuela de Enfermería, UCR: Distribución de opiniones del ejercicio de la \\ responsabilidad social ante la prevención de drogas, según número de respuestas *
}

Enunciados $\quad \frac{\text { Respuestas }}{}$

No ejercemos, porque no nos interesamos en conocer las leyes y somos individualistas

Lo mejor es hacerlo en forma anónima, porque desgraciadamente hay demasiada corrupción

No. Por miedo, porque nos sentimos solos y nos da miedo las represalias

No todos, porque no solo desconocemos la ley sino que no asumimos el problema como nuestro

Total

40

Fuente: Propia

*P. Según la Constitución de Costa Rica, las personas no pueden alegar desconocimiento de la ley, por lo tanto, es necesario conocer los deberes que se tiene como ciudadanos. A pesar de ello, ¿ejercen los ciudadanos su responsabilidad ante la prevención de drogas? ¿Por qué?

Dentro de la información recolectada, se revela que las personas encuestadas, en su totalidad, consideran que en Costa Rica no se ejerce la responsabilidad ciudadana para contribuir a la prevención en lo que respecta al fenómeno de las drogas, no obstante, se reconoce la información que poseen sobre acciones factibles para prevenir el fenómeno de las drogas.

Por otra parte, se analizó la percepción de los participantes en cuanto a la prevención, así como las áreas que consideran efectivas en lo que respecta a la temática y el reconocimiento de acciones que pueden realizarse de manera personal; en relación con lo anterior, se contó con el aporte de un total de 58 personas: 44 coincidieron en que las acciones que puede llevar a cabo un ciudadano, para prevenir el fenómeno de las drogas, consideran el fomento de valores como la unión familiar, el estudio o trabajo, evitar la vagancia, así como cuidar las amistades.

Del total correspondiente a este rubro, 20 personas afirmaron que la educación es un factor necesario para desarrollar la responsabilidad ciudadana; de igual forma, cuatro personas indicaron que se debía incluir valores espirituales, amor y respeto en la educación de los hijos, mientras que otro grupo, formado también por cuatro integrantes, mencionó la educación en la familia y la búsqueda de la felicidad. 
Es válido explicar que la mayoría de la población conoce parcialmente qué aspectos debe manejar, mejorar o tomar en cuenta para prevenir el consumo de drogas; sin embargo, los enfocan principalmente en acciones dentro del núcleo familiar, de modo que dejan de lado muchos otros elementos que los ciudadanos pueden desempeñar en el nivel comunitario, como se muestra en el gráfico 1.

\section{Gráfico1}

\section{Acciones que puede realizar un ciudadano para la prevención de las drogas}

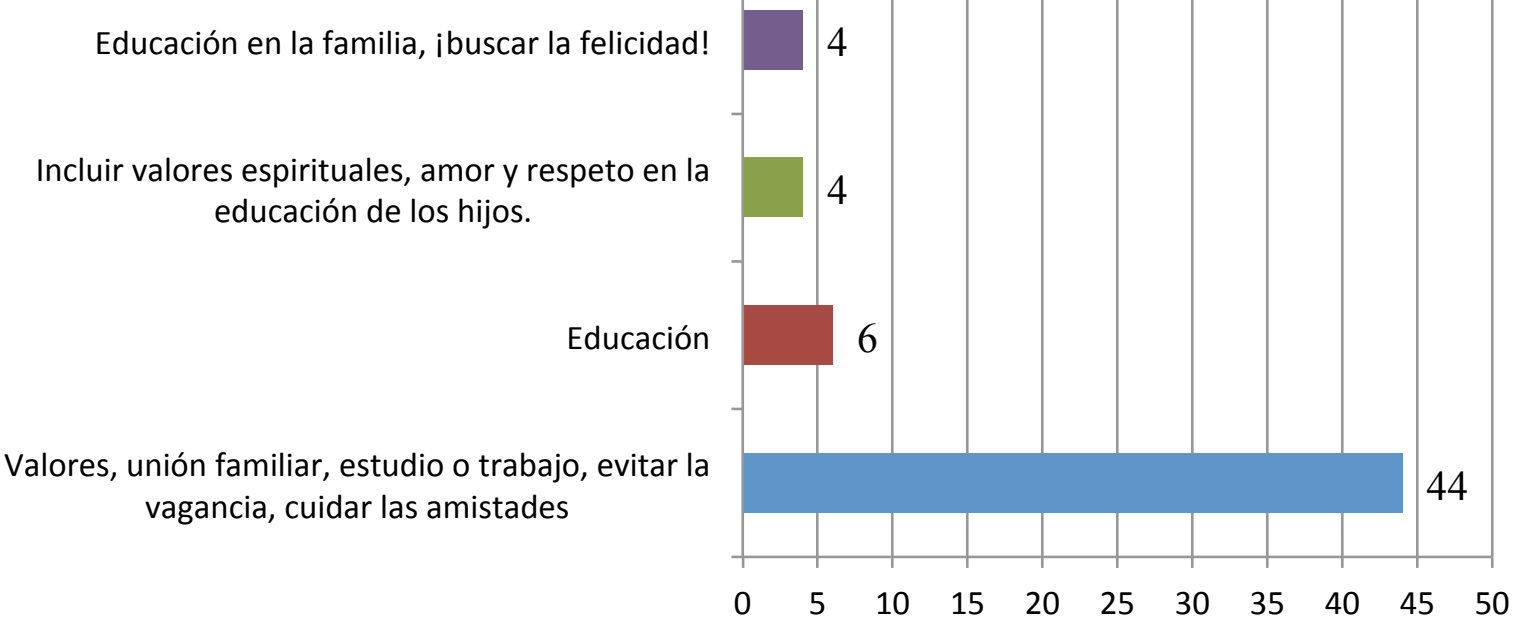

Fuente: Propia.

La mayoría de personas (43) mencionaron como parte de la responsabilidad de cada individuo en la prevención de drogas el denunciar a quienes las venden que, aunque no corresponde a la acción más importante dentro de la prevención, y su finalidad no es mitigar los daños producidos por este fenómeno, puede considerarse como parte de la prevención, de manera indirecta. Por otra parte, 13 de los participantes incluyeron aspectos como la promoción de ambientes seguros en la comunidad.

Finalmente, una parte de los encuestados expresó que cada quien es responsable de sus actos, que no se tiene ninguna responsabilidad ciudadana de cara a las drogas, lo cual refleja una posición de apatía e individualismo en relación con problemáticas sociales.

El concepto de responsabilidad ciudadana, su ejercicio, las acciones y los factores que la promueven o debilitan son parte de los elementos mencionados por la población participante en lo que respecta al fenómeno de las drogas, lo cual permite conocer la realidad de una sociedad inmersa en un sistema y analizar el tema de cara a la prevención de dicho fenómeno. 
Como se observa en el gráfico 2, a partir de la percepción de los encuestados, más de la mitad considera que las personas tienen el mismo nivel de responsabilidad en cuanto al ejercicio de la ciudadanía, sin que intervenga el factor económico en ello; de modo que, debido a las distintas leyes, regulaciones e, incluso, valores sociales, todo ciudadano tiene la misma obligación de cumplir con sus deberes, sin importar el aspecto económico.

Sin embargo, el resto considera que la condición económica sí es un factor que influye de manera total o parcial en el rol de cada persona como miembro de una sociedad, al punto de que la consideran un determinante significativo para el ejercicio de la responsabilidad ciudadana.

\section{Gráfico 2}

\section{Numero de respuestas según factibilidad ante la prevención del fenómeno de las drogas}

De eso dependerá el rol y comportamiento dentro de un grupo social.

Claro, es un factor que va influir en el rol de cada persona como cuidadano.

No, todos (as) somos responsables independientemente del factor económico.

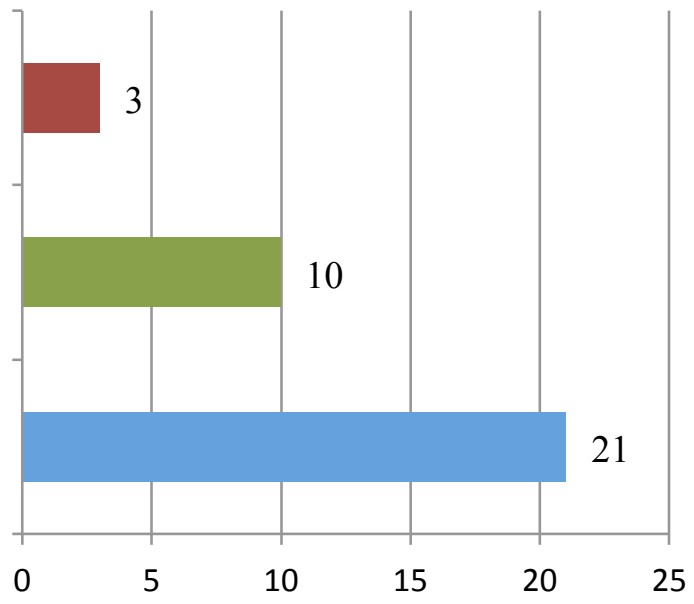

Fuente: Propia

Se puede afirmar que la mayoría de la población consultada considera que el fenómeno de las drogas no puede ser prevenido y que la solución parcial es la toma de medidas compensatorias y estabilizadoras; es decir, si el grupo habla de incapacidad de prevenir dicho fenómeno, sus acciones probablemente irán dirigidas en otra línea distinta de la prevención.

\section{DISCUSIÓN}

Los datos obtenidos del estudio reflejan que el espacio en la red social Facebook fue mayormente seguido y utilizado por mujeres en etapas de adolescencia y adultez temprana, lo cual evidencia una muestra cualitativamente representativa de las personas que la, lo cual concuerda con lo dicho por Caldevilla (2010), cuando comenta que dentro de las encuestas realizadas en Estados Unidos, el "usuario medio es una joven de entre 18 y 34 años” (p. 61), es decir, en su mayoría se refiere a mujeres en la etapa de adultez temprana. 
Respecto de lo anterior, Sánchez, Recio, Villegas y Olivera (2009), mencionan que la gente joven es la que mejor se ha sintonizado con las redes sociales, en donde buscan una nueva forma de comunicarse, intercambiar datos, experiencias y formalizar nuevas actitudes frente a la sociedad.

En cuanto a la variable de responsabilidad ciudadana, como se observó en la sección de resultados, la mayoría de los participantes concuerdan en que la responsabilidad ciudadana compete a las obligaciones que cada persona tiene como miembro de una sociedad, postura homóloga a la Escámez y Gil (2001), quienes afirman que

el ciudadano es miembro de una comunidad política, que le reconoce y protege como sujeto de derechos, mientras que él está obligado a cumplir las leyes o normas de esa comunidad e invitado moralmente a adquirir y practicar las competencias personales, profesionales y cívicas para el mantenimiento y mejora de la misma (p.37).

Sin embargo, como lo menciona King (citado por Leiva y Rojas, 2008), como ser social, cada persona es libre de elegir, de asumir su sistema de valores y su verdad, por lo que una formación desde la cultura de responsabilidad representa una herramienta de gran importancia, cuando el propósito es que el individuo se apropie dicho valor, considerando además que promoverá el cumplimiento de su rol dentro de un grupo social, definido por la cultura en la que se encuentre inmersa la persona. Por ende, para alcanzar una verdadera integración de los derechos y deberes de los ciudadanos, es esencial fortalecer el vínculo entre la comunidad y sus miembros y viceversa, dado que las adaptaciones a la vida y salud están influidas por las interacciones del individuo con su entorno, de modo que, como lo explica King (1984), el modo en que los seres humanos interaccionan con este es esencial para mantener su salud (citado por Leiva y Rojas, 2008). En otras palabras, para proteger los derechos, el individuo debe, además de defenderlos, aceptar sus responsabilidades en la sociedad; mientras que la comunidad, por medio de las instituciones y actores sociales, velará por el respeto de estos derechos y el cumplimiento de las leyes.

Por otra parte, en cuanto al ejercicio de la responsabilidad respecto de prevenir el fenómeno de las drogas por parte del ciudadano, se encontró que no es ejercida, principalmente por desconocimiento de las leyes e individualismo, según mencionan las personas participantes. Una de las posibles causas de la presencia de este desinterés en la población se refiere al "síndrome de casa ajena" definido por Campos (2010) como el deterioro de la ciudadanía, aunado a la exacerbación del individualismo costarricense., el cual se expresa mediante dos aspectos: uno, con intenciones de crítica social y otro, que consiste en la predisposición a soluciones egoístas e, incluso, antisociales.

Lo anterior se sustenta con el estudio realizado por Jiménez (2008) en el cual menciona que la incapacidad de los Estados de dar una respuesta oportuna y democrática a los pedidos de seguridad por parte de los ciudadanos, ha llevado a la pérdida de credibilidad de los habitantes en sus propios gobiernos, y al incremento de la ilegitimidad de las instituciones. A partir de ese momento, cada vez es más común escuchar términos como "ingobernabilidad" y "rompimiento del contrato social", situaciones en las que muchos tratan de tomar la solución por sus propios medios, al margen de la ley, lo cual agrava el problema. 
Tal como evidencia el párrafo anterior, sentimientos de temor y apatía culminan en un poco ejercicio de la responsabilidad por parte de la ciudadanía, lo que responde a una serie de eventos que marcan la cultura y el ejercicio de esta.

Las diversas acciones del Estado que concluyen en situaciones sin éxito, traen como consecuencia un detrimento de la confiabilidad de la población, la cual pierde interés por conseguir -mediante los entes rectore-s el apoyo para tratar problemáticas que afectan las mismas. Por tanto, las acciones que cada persona considere factibles en cuanto a participar de manera activa en la búsqueda de soluciones para los problemas sociales, permiten identificar un marco para la responsabilidad ciudadana, aunque la evidencia muestre lo contrario.

Efectivamente, la indiferencia por el ejercicio de la responsabilidad ciudadana no puede ser observada únicamente como una actitud poco consciente en relación con una realidad evidente como es el fenómeno de las drogas, sino como muestra de aspectos que influyen no solo en la visión de la realidad social, sino como determinantes de la salud.

A pesar de que las personas afirman no ejercer su responsabilidad como ciudadanos en lo que concierne a la problemática de las drogas, es necesario enfatizar el conocimiento que poseen sobre acciones factibles para prevenir el fenómeno de las drogas, entre las que mencionaron la educación en la familia, el fomento de valores y el estudio y/o trabajo.

Respecto de lo anterior, Bastardo y Genao (2001) afirman que es en la familia donde el individuo aprende a interaccionar con los demás, comienza el proceso de socialización y asimila las normas y los valores que lo formarán como persona; es decir, se le considera la primera escuela, lugar en el que el sujeto moldea su personalidad. De acuerdo con lo anterior, los participantes de la investigación reconocen que el proceso de socialización representa la base de cada individuo para la formación de una cultura de responsabilidad en cuanto a la prevenir el fenómeno de las drogas.

Además, cabe resaltar el papel que se les atribuye a las escuelas, puesto que según los resultados, toda la educación que se brinda en los hogares debe ser reforzada, de modo que se forme al individuo como un ser responsable de los asuntos no solo personales, sino también de la comunidad: es un trabajo en conjunto de la familia y las instituciones educativas. En otras palabras, estas pequeñas comunidades ejercen un papel importante de cara a la educación de los sujetos, por lo tanto, propician el desarrollo de valores que fomenten la cultura individual de la responsabilidad, así como la unión entre los ciudadanos para exigir el respeto a sus derechos.

Tales acciones son de gran relevancia para el fortalecimiento de esta cultura de responsabilidad, ya que como lo afirman García y Sánchez (2005), es necesario insistir en la labor de la educación para la salud, tanto en la escuela como fuera de ella para enfrentar la realidad de este fenómeno, con el objetivo de formar personas y ciudadanos que promuevan y promocionen actitudes, valores y habilidades que favorezcan un equilibrio saludable que aumente la calidad de vida. Sin embargo, pese a que la población reconoce estas acciones como medidas preventivas factibles, al referirse a sus obligaciones en cuanto al tema, como se notó en el apartado anterior, tan solo mencionan como medida principal la denuncia de casos. 


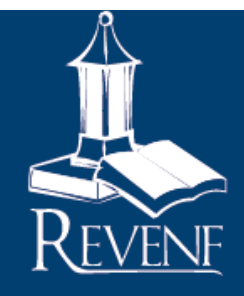

\section{Revista Electrónica Entermeria actual en costa Rica}

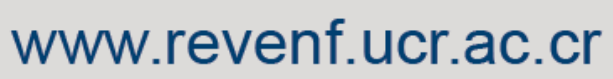

Por otra parte, en lo que concierne a los factores que intervienen en el ejercicio de la responsabilidad ciudadana en cuanto a la prevención de drogas, la mayoría de los participantes no considera que la situación socioeconómica influya en este tópico. No obstante, dicha situación socioeconómica es parte de los determinantes que producen inequidad en la salud, lo cual afecta sobre todo a los grupos más oprimidos y explotados de la sociedad. Lo mencionado tiene también un componente geográfico, puesto que, como lo explican Benach y Muntaner (2008), dichas clases sociales viven muchas veces en barrios «marginados» y son explotados, excluidos y oprimidos por la sociedad de múltiples maneras, debido a que tienen menos recursos económicos y poder político, peor atención sanitaria y servicios sociales, y están más expuestos a factores de riesgo dañinos para la salud, ya sean estos de tipo social, laboral o ambiental.

Asimismo en cuanto a la responsabilidad social que debe asumir el sector privado, en cuanto a prevenir dicho fenómeno, según la percepción de las personas participantes, este tiene responsabilidades con la sociedad, temática también señalada por el Centro de Información del Instituto de Normas Técnicas de Costa Rica (INTECO, 2011), entidad que aclara que en Costa Rica existe la norma INTE-ISO 26000:2010, la cual es una guía internacional sobre la responsabilidad social que se recomienda a las empresas ejercer. Sin importar sus características, la norma pretende orientar acerca de las áreas en las cuales se debe enfocar la responsabilidad social, considerando las particularidades que cada contexto exige y, obviamente, la participación en los diferentes espacios para la prevención, lavado de dinero, etc., que el fenómeno de drogas genera, acciones sobre las que el sector privado tiene mucho que aportar.

En relación con otros temas, fue significativo identificar la percepción general de la población en cuanto a la factibilidad de prevenir las drogas, aspecto acerca del que consideran que el fenómeno de las drogas no puede ser prevenido y que la solución parcial es la toma de medidas compensatorias y estabilizadoras, es decir, si el grupo habla de la incapacidad de prevenir dicho fenómeno, sus acciones probablemente se dirigirán en una línea distinta a la de la prevención. Acerca de este punto, es vital mencionar que la salud y la prevención de fenómenos como el de la drogadicción, contienen una serie de aristas que abarcan una gran cantidad de temáticas, dentro de las que se destaca la prevención que, según, Jiménez, Morante, Lozada, Rodríguez y Garrosa (2004) es,

Concienciar, no imponer o meramente institucionalizar ciertas medidas de actuación. Se ha de intervenir capacitando, no simplemente marcando límites... Prevenir en materia de drogas, en fin, ha de ser un acto de mediación, más que de imposición y de persuasión represiva o de sanción correctiva. Prevenir es también intervenir, aun cuando no en toda intervención se plantea como fin último un acto preventivo. (p. 25)

En la participación activa de la ciudadanía es fundamental incluir la salud en materia de prevención es decir, se debe constituir en un acto de mediación, en el cual se realice un trabajo en conjunto, con la finalidad de prevenir de la mejor manera situaciones como la drogadicción y todas sus repercusiones en la sociedad (Jiménez, 2008).

Por su parte, Bejarano (2006) propone que para prevenir el consumo de drogas, se necesita un conjunto de intervenciones que permitan fortalecer los factores de protección y disminuir los de riesgo, afirmación que destaca la importancia de reconocer las acciones posibles que toman las personas, en su condición de ciudadanos y ciudadanas, para prevenir fenómenos como este. 
Asimismo, Gómez y Guitart citados por Sánchez (2004). afirman que la construcción de valores debe enlazar los de la comunidad con el propósito de formar individuos capaces de enfrentar problemáticas sociales y personales, mediante actitudes que fortalezcan su seguridad personal. A raíz de esto es evidente que los valores constituyen un pilar fundamental en la formación de ciudadanos que ejerzan de manera responsable su rol en la sociedad, participando activamente en el mejoramiento de la salud pública y personal, así como en la solución de problemas sociales, como lo es el caso del fenómeno en estudio.

Sin embargo, para fortalecer los valores, es imprescindible que se trabajen desde la familia, tal como lo reconoce la población participante, debido a que el núcleo familiar es la primera célula social, en la cual comienza la construcción del yo que nos identificará más adelante como personas; es el espacio donde la persona aprende a interaccionar con los demás y se da, por consiguiente, el llamado proceso de socialización, mediante el que asimila las principales normas y valores para el resto de su vida (Bastardo y Genao, 2001).

Cabe resaltar que los valores son una respuesta mencionada por los participantes en distintas ocasiones, así como la educación, dado que ambos son vitales para que un individuo ejerza su rol ciudadano, con todas las obligaciones que conlleva. Tal como lo explica Cuadra (2001), si todos somos responsables de nuestros actos, sin decir que simplemente seguimos órdenes, comprenderemos que es necesario educar para analizar, discurrir, decidir, o sea, educar para la responsabilidad y el diálogo.

Siguiendo esta línea, la población participante, al hacer énfasis en los valores y la educación, coinciden también con Bastardo y Genao (2001), quienes afirman que el término prevención "es y ha sido usado de varias formas, pero cualquiera de ellas acepta que el prevenir es hacer algunas cosas ahora, antes que un problema aparezca" (p. 31).

Junto a esta consideración de hacer algo antes de que ocurra, también se puede hacer mención de la necesidad de promover condiciones que reduzcan al mínimo los riesgos, en cuanto a prevalencia e incidencia de problemas sociales, trabajar desde las comunidades con actitudes positivas y soluciones en conjunto que permitan la consecución de metas preestablecidas para el beneficio del espacio comunal.

A partir de lo mencionado, se puede deducir que la población del estudio conoce el concepto de prevención y menciona diferentes maneras de cómo llevarla a cabo: rescatando los valores, mediante la convivencia familiar, evitando el ocio y fortaleciendo la educación. Sin embargo, no se puede concluir que la ciudadanía esté dispuesta a realizar esas acciones, ni tampoco si tiene las condiciones necesarias para ejecutar las que ellos mismos mencionan.

Como lo menciona la población en estudio, es necesario destacar que, el fenómeno de las drogas, como problema de salud pública, debe ser enfrentado tanto por el gobierno, como por organizaciones, instituciones, asociaciones, comunidades, familias e individuos; es decir, es una responsabilidad de todos.

La información obtenida en este trabajo en cuanto a la participación y responsabilidad ciudadana, no debe tomarse necesariamente como una muestra de irresponsabilidad o falta de civismo, sino como la falta de participación individual en asuntos que competen al colectivo, de manera directa o indirecta, ya que en gran 
medida los participantes se ven inhibidos por razones como temor, incredulidad en la política pública y desconocimiento de las leyes, sobre los que debe trabajarse desde los profesionales del área de la salud.

\section{CONCLUSIONES}

La población del estudio reconoce la responsabilidad ciudadana como una obligación o compromiso que cada individuo tiene con la sociedad a la que pertenece.

Se evidencia desconocimiento y confusión en cuanto a la responsabilidad de los ciudadanos en la prevención de las drogas, puesto que los participantes únicamente mencionan la denuncia como medida para ejercer tal responsabilidad.

La totalidad de la población de estudio coincide en que las organizaciones de índole privado en Costa Rica tienen responsabilidad con la sociedad en lo referente a la prevención del fenómeno de las drogas.

Parte de los costarricenses no ejercen su responsabilidad como ciudadanos y ciudadanas, principalmente por desconocimiento, sea de leyes o de políticas públicas.

Parte de la población participante delega gran parte de sus responsabilidades como ciudadanos a diferentes entes, personas o instituciones.

Los participantes reconocen la importancia de las acciones que se pueden y deben realizar en el núcleo familiar, para la prevención del fenómeno de las drogas y el fomento de la responsabilidad ciudadana en cuanto a este tema se refiere.

Del trabajo se desprende que la mayoría de la población participante, reconoce como acciones para la prevención de las drogas principalmente el fomento de valores, el estudio o trabajo, y la educación en la familia.

Los profesionales de enfermería pueden ser líderes en cuanto a la educación y el empoderamiento de las personas en cuanto a su responsabilidad de cara a la prevención de las drogas, puesto que esta es una profesión con visión social que tiene la facilidad de acceder e influir en la ciudadanía.

\section{REFERENCIAS BIBLIOGRÁFICAS}

Bastardo, D.,Genao M. (2001) Manual para la prevención del uso indebido de drogas en la comunidad. Santo Domingo: Editorial de Colores.

Beck, U. (2007). La Sociedad del Riesgo Mundial. (2a ed). Madrid: Paidós.

Bejarano, J. (2006). Inseguridad ciudadana y drogas. Realidades y percepciones. San José: Lara Segura y Asociados.

Benach, J., Muntaner, C. (2008). Red de condiciones de empleo y desigualdades en salud(Emconet): informe global sobre la desigualdad en salud. Toronto: OMS. 
Blázquez, N. (2004). La bioética y los hijos del futuro. Madrid: Editorial Visión NET.

Caldevilla, D. (2010). Las redes sociales. Tipología, uso y consumo de las redes 2.0 en la sociedad digital actual. España: Documentación de las ciencias de la información. $\mathrm{N}^{\mathrm{0}} 33$.

Campos, A. (2010).Violencia Social. San José: Editorial EUNED.

Constitución Política de Costa Rica.

ehttp://pdba.georgetown.edu/Parties/CostaRica/Leyes/constitucion.pdf

(1949). Recuperado

de:

Consejo de Organizaciones Internacionales de las Ciencias Médicas (CIOMS) y Organización Mundial de la Salud (2002). Pautas éticas internacionales para la investigación biomédica en seres humanos. Ginebra: OMS.

Cortina, A. (1997). Ciudadanos del mundo: hacia una teoría de la ciudadanía. Madrid: Editorial Alianza.

Cuadra, A. (2001). Cómo informar cultivando y promoviendo la responsabilidad ciudadana. Canarias, España. Revista Latina de Comunicación Social. 4. $\mathrm{N}^{\circ} 41$.

Dirección Triángulo de Solidaridad. (2000). Ética y valores para una ciudadanía activa. Costa Rica. Ministerio General de la República.

Escámez, J. y Gil, R. (2001). La educación en la responsabilidad, Barcelona: Paidós.

Flores, J. (2009).Nuevos modelos de comunicación, perfiles y tendencia en las redes sociales. España. Revista Científica de Educomunicación, 17. n 33. p. 73 - 81.

Gamboa, L. (2007). Los retos de la ciudadanía y la gobernabilidad en la globalización. (1er ed). San José: Editorial UCR.

García, A. y Sánchez, M. (2005). Drogas, sociedad y educación. España: Universidad de Murcia. Servicio de Publicaciones ON LINE.

Hernández, R., Fernández, C. y Baptista, P. (2007). Fundamentos de metodología de la investigación. Madrid: McGraw-Hill.

Instituto de Normas Técnicas de Costa Rica (2011). Norma INTE-ISO 26000:2010. Costa Rica: INTECO.

Jiménez, F. (2008). Análisis del antecedente familiar de delictividad y la historia personal de consumo de sustancias piscoactivas, en los menores de edad infractores de la ley, privados de libertad en Costa Rica, como factores de vulnerabilidad para su conducta delictiva. Proyecto Final de Graduación Para optar por el título de máster en Criminología y Derechos Humanos. San José, Costa Rica. Universidad para la Cooperación Internacional. 
Jiménez, B., Morante, M., Lozada, M., Rodríguez, M., Garrosa, E. (2004). El estrés traumático secundario, evaluación, prevención e intervención. Santiago: Sociedad Chilena de Psicología clínica.

Leiva, V. , Rojas, L. (2008). Material parcial del módulo de autoinstrucción de teorías de enfermería. San José: Editorial Universidad de Costa Rica.

Ministerio de Salud de Costa Rica. (1973). Ley general de Salud de Costa Rica. San José: La Gaceta No. 222.

Organización Mundial de la Salud. (2000) Informe sobre la salud en el mundo 2000 - Mejorar el desempeño de los sistemas de salud.- Ginebra: OMS.

Organización Mundial de la Salud (2006). Informe de situación general de salud. Ginebra: OMS.

Sánchez, F. (2004) Construcción de valores en salud. Rev. Costarricense de salud pública. 13, (25) 01-11. Recuperado de: <http://www.scielo.sa.cr/scielo >.

Sánchez, J., Recio, J., Villegas, R., Olivera, M. (2009). Aspectos legales y documentales de las redes sociales: el modelo Facebook. Ibersid, 187-195. Recuperado de: http://www.ibersid.eu/ojs/index.php/ibersid/article/viewFile/3739/3500 